\title{
Manifestations of Geometry in Architecture
}

\author{
Kim Williams $^{1}$ (D) Michael J. Ostwald ${ }^{2}$
}

Abstract The Co-Editors-in-Chief of the Nexus Network Journal introduce the contents of vol. 19, no. 1 (2017).

Keywords Architectural design · Design concepts · Archaeoastronomy · Fractal dimensions - Gothic architecture - Traditional Iranian brick patterns $\cdot$ Golden mean

Geometry and architecture have been linked from the earliest times when humans began to build structures based on codified information, that is, not simply piling or heaping blocks of stone or pieces of timber one on top of another, but assembling those materials together in a way that was in accordance to a pre-conceived notion of a finished artefact. This first issue of vol. 19 (2017) of the Nexus Network Journal contains a number of research papers that examine various manifestations of geometry in buildings that range from the ancient to the contemporary.

In an architectural context, geometry is often used as an ordering device, that is, a way to link spaces to functions. In 'The Spatial Logic of Mamluk Madrassas: Readings in the Geometric and Genotypical Compositions', Shatha Malhis of Jordan examines the geometric and spatial attributes of educational buildings (Madrassas) built during the Bahri and Burji periods (1260-1517 A.D.). Malhis's

$\triangle$ Kim Williams

kwb@kimwilliamsbooks.com

Michael J. Ostwald

michael.ostwald@newcastle.edu.au

1 Kim Williams Books, Corso Regina Margherita, 72, 10153 Turin, Italy

2 School of Architecture and Built Environment, The University of Newcastle, University Drive, Callaghan, NSW 2308, Australia 
paper highlights how the geometry and resulting spaces of these buildings varied between 'centripedal' and 'centrifugal'.

Once a structure is codified and built, geometry can add beauty to a design. Paradoxically, sometimes the greatest beauty comes from a breakdown of order. Emil Makovicky of Denmark and Nicolette M. Makovicky of London, in 'Nonperiodic Octagonal Patterns from a Jali Screen in the Mausoleum of Muhammad Ghaus in Gwalior and Their Periodic Relatives' illustrate the geometrical sophistication used to create nonperiodic eightfold patterns in the 1500 s in Northern India.

Geometry can provide strength as well as order, as shown by the form of the catenary arch. A team of Spanish researchers, composed of Josep Lluis i Ginovart, Agustí Costa-Jover, Sergio Coll-Pla and Mónica López Piquer, have investigated the use of catenary arches from the eighteenth century through the twentieth. In 'Layout of Catenary Arches in the Spanish Enlightenment and Modernism' they show how the geometry of the chain-shaped form evolved from practical uses in gunpowder magazines to a refined form of architectural expression.

The word 'geometry' is derived from the Greek roots geo- (earth) and -metron (measurement), but knowledge of geometry extended far beyond what was known about the planet to embrace the larger cosmos in which it exists. There were practical implications of the relationship between the planet and the heavens. For example, shadows cast by the sun allowed for the measurement of time. In 'Geometry and the Restoration of Ancient Sundials: Camera Obscura Sundials in Cava de' Tirreni and Pizzofalcone' Italian scholars Alessandra Pagliano, Angelo Triggianese and Luca Santoro show how the geometry of 'camera obscura' sundials can be tweaked in order to restore historic but neglected sundials to working condition.

Of course, in order to concretise abstract relationships between geometry and architecture, a precise method of representation is needed, and over many centuries architects developed and refined systems of technical drawings to achieve this. The origins of representation go very far back indeed, with Adriana Rossi investigating a potential example from at least 5000 years ago. In 'The Origin of Technical Drawing in the Narmer Palette' Rossi shows how a tiny glyph on this ancient Egyptian artefact can be read as a technical drawing of a fortified city wall.

Geometry is as valuable a tool for form-making today as it was in ancient times, and we have many more instruments at our disposal for its exploitation. Iranian architect Shaghayegh Rajabzadeh and Italian engineer Mario Sassone teamed up to develop a computer modelling approach to modern-day brick patterning, capable of defining the brick courses, handling mortar thickness, and controlling all the related geometrical issues of free-form surfaces. Their results are presented in 'Brick Patterning on Free-Form Surfaces'. In 'Robotic Technologies for Non-Standard Design and Construction in Architecture', José Pedro Sousa, director of the Digital Fabrication Laboratory at the University of Porto's Faculty of Architecture, illustrates the ways in which today's technology supports geometric freedom and mass customization.

Finally, geometry generates proportion. In 'Ratios from the Intersections of $10+1$ Proportionalities', Hyoung-June Park of the University of Hawaii at Manoa 
presents the results of an analysis of the proportional ratios for rooms preferred by Vitruvius, Alberti, Serlio and Palladio. Such room ratios are of course derived from geometric shapes: squares and rectangles. Then comparing these historically cited ratios with those analysed by Fechner and Lalo several hundred years later, Park shows that all of these can in fact be reduced to a small set of universally sanctioned ratios. In 'Geometrical Proportion in the Sixteenth Century: Methods and Constraints' José María Calvo-López and Macarena Salcedo-Galera have analysed the geometrically-generated proportions in a sixteenth-century treatise attributed to Spanish architect Rodrigo Gil de Hontañón to show that geometry can lead to both commensurate and incommensurate ratios, and that it is possible to find in theories about Spanish sacred architecture both rational and non-rational proportions.

In this issue's contribution to the 'Geometer's Angle' column, California-based artist and geometer Mark Reynolds beautifully illustrates how proportional systems are derived directly from geometrical exploration. In 'Marriages of Incommensurables: $\phi$-Related Ratios Joined with $\sqrt{ } 2$ and $\sqrt{ } 3$ ', his step-by-step geometrical constructions are flanked by some of his own artworks based on these findings.

In this issue, Filippo Fantini reviews Mark Wilson Jones's book, The Origins of Classical Architecture: Temples, Orders and Gifts to the Gods in Ancient Greece. Of special interest in this book is the fifth chapter, which deals with the geometricalmathematical foundations of Greek temples.

This issue is completed by three conference reports on recent international meetings whose scope includes geometry. The proliferation of such meetings shows geometry's continued relevance for today's makers of architecture. Micaela Colella reports on 'Structures, Algorithms and Stone/Timber Prototypes', which took place in Bari in January 2016. Paul Gailiunas reports on 'Bridges Jyväskylä 2016', the nineteenth edition of the ongoing 'Bridges' conference series on mathematical connections in art, music, architecture, education, and culture. Orkun Kasap reports on 'Advances in Architectural Geometry (AAG) 2016 Symposium', the fifth edition of a conference series devoted to the specialised discipline of architectural geometry.

Kim Williams is the founder and Co-Editor-in-Chief of the Nexus Network Journal: Architecture and Mathematics. She also founded and directs the international conference series 'Nexus: Relationships Between Architecture and Mathematics.

Michael J. Ostwald is Professor and Dean of Architecture at the University of Newcastle, Australia. He is a Visiting Professor at RMIT University and a Research Fellow at SIAL (Melbourne). He has held past academic positions in the USA and Hong Kong and worked for architectural practices in Australia, the UK and the USA. Michael has a PhD in architectural theory and history and a higher doctorate (DSc) in design mathematics. He is Co-Editor-in-Chief of the Nexus Network Journal: Architecture and Mathematics (Springer) and a member of the Editorial Boards of $A R Q$ (Cambridge) and Architectural Theory Review (Taylor \& Francis). 